\title{
Artigos
}

\section{Educazione all'arte come esperienza, comunicazione, sperimentazione e documentazione: Un percorso di ricerca in Italia nei servizi educativi}

\begin{abstract}
Riepilogo: Questo scritto vuole riflettere sul ruolo dell'educazione all'arte nei contesti educativi italiani, in particolare riferendosi alle proposte pensate per i bambini più piccoli. A partire da una rapida panoramica relativa all'evoluzione della parte dell'educazione all'arte nei programmi delle scuole elementari (6-10 anni) realizzata per comprendere il pensiero educativo a questi sottostante, il contributo si muove ad analizzare tre tematiche sentite come attualmente rilevanti. La prima legata alla connessione con il mondo dell'arte contemporanea, con il suo portato politico di comunicazione; la seconda che analizza l'importanza dell'esperienza laboratoriale, nel senso di allestimento degli spazi e di predisposizione dei materiali, con le sue possibilità legate alla sperimentazione; la terza che si sofferma sulla potenzialità della documentazione come forma di riflessione metacognitiva sia per i bambini, sia per gli adulti, educatori, responsabili dei servizi e genitori.
\end{abstract}

Parole chiave: Educazione all'arte. Arte contemporanea. Laboratorio. Esperienza. Sperimentazione. Documentazione. Comunicazione. Musei.

\section{Educação pela arte como experiência, comunicação, experimentação e documentação: um percurso de pesquisa na educação infantil na Itália.}

\begin{abstract}
Resumo: Este artigo tem como objetivo refletir a respeito do papel da educação pela arte nos contextos educacionais italianos, em particular referente às propostas destinadas as crianças pequenininhas. A partir de uma breve panorâmica relativa a evolução da parte da educação pela arte nos programas das escolas "elementares" (6-10 anos), realizada para compreender o pensamento educacional que a sustenta, este artigo analisa três temáticas compreendidas atualmente como relevantes. A primeira ligada à conexão com o mundo da arte contemporânea, com o seu alcance político de comunicação; a segunda, que analisa a importância da experiência em oficinas no sentido da organização dos espaços e da predisposição dos materiais com as suas possibilidades relativas à experimentação; a terceira, que se foca na potencialidade da documentação como forma de reflexão meta-cognitiva tanto para as crianças, quanto para os adultos educadores, gestores das unidades educativas e pais e mães.
\end{abstract}

Palavras-chave: Educação pela arte. Arte contemporânea. Oficinas. Experiência. Experimentação. Documentação. Comunicação. Museus.Educação Infantil.

\section{Art education as experience, experimentation, communication and documentation: A research path in Italy in early childhood education/ educational services}

Abstract: This paper wants to reflect on the role of art education in the Italian educational contexts, particularly referring to the proposals designed for younger children. Starting from a quick overview of the evolution of the part of art education in

1 Università Milano-Bicocca. Dipartimento di Scienze Umane per la Formazione. Docente di Didattica Generale ed Educazione all'Immagine. E-mail: franca.zuccoli@unimib.it 
the elementary school (6-10 years) programs designed to understand the underlying educational thinking, the contribution tries to analyze three issues that are currently relevant. The first linked to the connection with the world of contemporary art, with its political reach of communication; the second that analyzes the importance of laboratory experience, in terms of space layout and material predisposition, with its experimental possibilities; the third one focuses on the potential of documentation as a form of metacognitive reflection for both children and adults, educators, service providers, and parents.

Keywords: Art education. Contemporary art. Laboratory. Experience. Experimentation. Documentation. Communication. Museums. Early childhood education

Educazione artistica, educazione all'immagine, arte ed educazione. Dal punto di vista dell'evoluzione storica

$\mathrm{P}$ er approfondire la tematica relativa all'arte in Italia, rapportandosi ai/alle bambini/e più piccoli/ $\mathrm{e}^{2}$, bisogna necessariamente fare riferimento anche all'evoluzione della scuola elementare ${ }^{3}$, dal $2003^{4}$ denominata primaria, cogliendo così i passaggi concettuali che hanno caratterizzato questa presenza. Si tratta, infatti, di un ambito di sapere complesso, costituito da innumerevoli fattori, tra i quali vale la pena di ricordare almeno: il mondo dell'arte, in cui quella a noi più contemporanea con le sue differenti azioni può avere un grande rilievo; la presenza dei media e dei materiali più attuali; la parte sostanziale legata alle specificità dell'espressione grafica e tridimensionale dei bambini; il rapporto con i patrimoni culturali e soprattutto gli " [...] interrogativi pedagogico-didattici relativi alle modalità con le quali sia possibile preparare e favorire un incontro significativo delle nuove generazioni con questo mondo [...]." (Mazza, 2001, p.7) Va segnalato, però, come non da subito l'arte e la sua educazione, abbiano avuto un ruolo definito all'interno dei programmi ministeriali. È necessario allora effettuare un, seppure veloce, viaggio all'interno della storia dei programmi educativi per meglio comprendere le scelte epistemologiche legate a questi cambiamenti. I programmi ministeriali risultano, infatti, degli ottimi strumenti per comprendere le prospettive culturali che i vari governi, che si sono susseguiti nel tempo, hanno ritenuto fondamentali per imprimere dei cambiamenti o per confermare lo status quo delle azioni educative messe in campo. Va però ricordato come la preoccupazione principale del nascente stato italiano nel 1861, data d'inizio di questa avventura, fosse quella di obbligare a utilizzare un'unica lingua nazionale, l'italiano, al posto dei dialetti, e di sconfiggere l'analfabetismo imperante che contava una media del $76 \% 5$.

2 Da questo momento, dopo aver specificato che il nostro pensiero considera sempre le bambine e i bambini si utilizzerà il maschile, come previsto dalla lingua italiana, per una comodità di scrittura.

${ }^{3}$ Si tratta del segmento scolastico che si occupa dei bambini dai 5 ai 10 anni. In Italia i servizi educativi da 0 a 3 anni si chiamano nido, dai 3 ai $5 / 6$ anni scuole dell'infanzia, dai 5/6 anni ai 10 scuola primaria, dai 10 ai 13 scuola secondaria di primo grado. Le Indicazioni nazionali del 2012 sono i programmi che si sono occupati delle scuole dai 3 fino ai 13/14 anni.

${ }^{4}$ II cambiamento del nome da scuola elementare in scuola primaria è avvenuto nel 2003 a seguito della riforma Moratti, legge 28 marzo 2003 n. 53.

${ }^{5}$ In alcune regioni, come la Sardegna, la media degli analfabeti era del $91 \%$, mentre in Calabria e in Sicilia era del $90 \%$. 
Analizzare la storia dei programmi dal punto di vista di una disciplina, in questo caso quella dell'educazione all'arte, ci permette di capirne la centralità o al contrario la marginalità o addirittura l'assenza, di conoscere il taglio culturale e le proposte operative che sono nate sempre a partire da un preciso posizionamento culturale. Se, infatti, osserviamo i programmi dal punto di vista peculiare dell'educazione artistica possiamo scoprire come i primi riferimenti proposti ci parlassero esclusivamente di attività legate esclusivamente al disegno, inteso come passaggio preparatorio all'apprendimento della scrittura e della bella grafia (calligrafia)

Solo nel 1894 riusciamo a trovare alcuni suggerimenti resi più espliciti, che si affidano alla "alla buona volontà e alla sagacia degli insegnanti $[\ldots]$ per educare l'occhio e la mano dei fanciulli $[. .$.$] e per$ rendere intuitivo e pratico il proprio insegnamento", si raccomanda di mantenere una giusta distanza dalla "[...] pretensione a formare piccoli artisti." Anche nei programmi del 1905 si parla solo di disegno, anche se maggiore è lo spazio che vi si dedica, differenziandolo nelle tipologie di: disegno libero, disegno geometrico, copia da modelli. Ecco alcune indicazioni che vengono fornite ai maestri: "[...] le forme e gli oggetti da ritrarre siano quelli che l'alunno conosce e ai quali si interessa, e il compito sia sempre proporzionato alla sua effettiva capacità."

Nei programmi del 1923 appaiono per la prima volta i concetti di espressione, spontaneità e si raccomanda agli insegnanti di non correggere, né di intervenire sui disegni dei bambini per non rischiare "l'uccisione della spontaneità dell'espressione grafica". Si propone ancora una suddivisione così articolata: "esercizi spontanei dello scolaro, disegno a memoria, disegno spontaneo dal vero."

Nel 1955 il titolo di un paragrafo Disegno e scrittura ci fa comprendere quanto il disegno fosse ancora legato a un uso funzionale. Sarà solo nei programmi del 1985 che l'Educazione all'immagine verrà per la prima volta considerata come una vera e propria disciplina con una sua autonomia. Con questa nuova proposta non ci si occupa esclusivamente di disegno, anzi l'azione si fa estremamente ricca, comprendendo: dal lavoro artigianale, alla televisione, dal fumetto, al patrimonio culturale, proponendo un'alternanza tra momenti di: fruizione, produzione e valorizzazione dei beni culturali. Il rapporto con il mondo dellimmagine è visto, dagli estensori dei programmi, come la possibile acquisizione e implementazione di un linguaggio complementare agli altri, da garantire nella sua specificità. Nella declinazione operativa, però, più che tratteggiare delle linee pragmatiche saldamente legate ai presupposti teorici, ci si riduce a un elenco di media e di tecniche da sperimentare con i bambini. Se velocemente ${ }^{8}$ arriviamo ai programmi attuali del 2012, chiamati Indicarioni naz̧ionali per il curricolo della scuola dellinfanzia e del primo ciclo d'istruzione, in cui già dal nome possiamo comprendere il taglio non più impositivo, ma propositivo, che implica una scelta, individuale dell'insegnante o collettiva di scuola, delle varie opzioni. Per quanto riguarda la disciplina Arte e Immagine troviamo alcune frasi significative che riteniamo

\footnotetext{
6 Per questo si vedano: Bettini, 1950; Lombardi, 1987.

7 In questa trattazione non sono analizzati i programmi redatti durante il regime fascista, che hanno come prima finalità quella di glorificare le azioni di Benito Mussolini, come pure quelli di Carleton Washburne scritti nel primissimo dopoguerra (1945) all'interno dei quali non si segnalano cambiamenti significativi.

${ }^{8}$ Anche in questo caso si è preferito non soffermarsi sui programmi del 2007 e del 2011, per la necessità di rispettare una trattazione sintetica. In ogni caso una grande traccia dell'elaborazione proposta da questi programmi si trova in quelli del 2012 a cui si dedica invece una maggiore attenzione.
} 
opportuno riportare in modo integrale. In particolare nella sezione dedicata alla scuola dell'infanzia intitolata Immagini, suoni, colori troviamo scritto così:

"I bambini esprimono pensieri ed emozioni con immaginazione e creatività: l'arte orienta questa propensione, educando al piacere del bello e al sentire estetico. L'esplorazione dei materiali a disposizione consente di vivere le prime esperienze artistiche, che sono in grado di stimolare la creatività e contagiare altri apprendimenti. I linguaggi a disposizione dei bambini, come la voce, il gesto, la drammatizzazione, i suoni, la musica, la manipolazione dei materiali, le esperienze grafico-pittoriche, i massmedia, vanno scoperti ed educati perché sviluppino nei piccoli il senso del bello, la conoscenza di se stessi, degli altri e della realtà." (Indicazioni nazionali, 2012, p. 20)

Proprio nei confronti dell'arte troviamo un paragrafo estremamente significativo che ci prospetta un'apertura non solo operativa, ma intesa in quanto linguaggio, possibilità, punto di vista, eccolo qui citato:

"L'incontro dei bambini con l'arte è occasione per guardare con occhi diversi il mondo che li circonda. I materiali esplorati con i sensi, le tecniche sperimentate e condivise nell'atelier della scuola, le osservazioni di luoghi (piazze, giardini, paesaggi) e di opere (quadri, musei, architetture) aiuteranno a migliorare le capacità percettive, coltivare il piacere della fruizione, della produzione e dell'invenzione e ad avvicinare alla cultura e al patrimonio artistico.” (Indicazioni nazionali, 2012, p. 20)

Per concludere questo rapido excursus storico e per comprendere quanto le proposte presentate abbiano risentito nel tempo del portato culturale legato alle diverse epoche e alle differenti prospettive politiche ed epistemologiche, e di come questo accada anche oggi, possiamo riferirci anche a un altro studioso, Arthur Efland. Egli ha studiato l'evoluzione storica dell'art education in particolare osservando in Europa: l'Inghilterra e la Germania, oltre al sistema scolastico americano, sottolineando la presenza di tre passaggi da lui così denominati differentemente. Si tratta del: filone espressivista per la presenza di una particolare attenzione al potenziale del soggetto, in cui l'attività artistica è intesa come gioco legato al sapere e al saper fare; filone costruttivista dove l'arte è vista come strumento formativo per una cittadinanza responsabile (Read, 1962); filone razionalista che la vede come una disciplina con specifici metodi d'indagine e di lavoro (Efland, 19909). L'appartenenza a un filone piuttosto che a un altro influenza in modo significativo le azioni didattiche che come educatore andrò a proporre, se, infatti, mi riferisco al filone espressivista darò una maggiore attenzione al soggetto che apprende, alle sue necessità personali, alla sua espressione e creatività, se mi riferisco al secondo filone costruttivista il mio obiettivo educativo sarà posto nella scelta di un apprendimento condiviso, sociale, collaborativo, nel terzo caso, il filone razionalista, la mia massima attenzione sarà dedicata alle specificità della disciplina, al suo corpus storico, contenutistico, linguistico, metodologico (Mazza, 2001, p.20).

In particolare nel percorso italiano l'uso delle discipline artistiche nei primi tempi è stato visto esclusivamente nella proposta del disegno, inteso come mezzo per facilitare l'apprendimento della scrittura, in un secondo momento il disegno ha previsto sia i contenuti realistici, sia la copia da modelli, sia il genere più astratto, per i più piccoli sono stati proposti anche materiali tridimensionali, sempre con un'alternanza tra possibilità spontaneistiche individuali e visioni più laboratoriali, sperimentali e sociali, per arrivare in ultimo verso un contatto con i nuovi media e con il patrimonio culturale, in un'ottica di attenzione e di valorizzazione.

\footnotetext{
9 Su questo lavoro hanno riflettuto numerosi autori, qui si citano almeno due riferimenti fondamentali: Siegesmund, 1998, pp.197-214;
} Mazza, 2001, pp.17-20. Interessanti anche le riflessioni di Swift (1999), sull'idea di un manifesto dedicato all'educazione all'arte. 


\section{Educazione all'arte come contatto con il contemporaneo}

Se ci si ferma però a una visione dell'arte in ambito educativo costretta in questi seppur interessanti parametri, ci si rende immediatamente conto di correre il rischio di rimanere all'interno di un'idea ristretta, limitata al disegno e a qualche sperimentazione, in cui il rapporto con la rappresentazione della realtà oggettiva risulta la parte preminente, facendo così scappare il portato innovativo, creativo che l'arte nel tempo ha sempre avuto e che oggi ha ancora di più.

Rivolgendosi alla scuola, infatti, anche dal punto di vista della sperimentazione di materiali e strumenti, molto spesso ci si trova di fronte, laddove questa sperimentazione venga proposta, a uno spontaneismo che non garantisce progressi nella conoscenza. "La produzione artistica degli alunni si presenta nella maggior parte dei casi stereotipata proprio perché, dietro alla cosiddetta spontaneità, non si intacca un convenzionale sistema di visione [...]" (Tornatore (a cura di), 1982, p.260). Fondamentale risulterebbe invece l'avvicinamento alla materia in modo strutturato (non inteso nel senso di preordinato, ma di esaminato cercando di valorizzare le massime potenzialità) e significativo, poiché “[...] la diretta manipolazione dei materiali svolge una funzione eminentemente cognitiva, qualora si approfondiscano tutte le possibilità intrinseche e strumentali del mezzo espressivo." (Tornatore (a cura di), 1982, p.260).

Con questo ritornando in pieno al confronto con l'arte contemporanea, fase in cui gran parte delle attuali sperimentazioni sono state realizzata e molte barriere tra: pittura, scultura, decorazione, architettura, musica, danza, movimento... sono state abbattute. Nell'arte di questo periodo l'attenzione verso il lato materiale e sperimentale della creazione è divenuta dunque un aspetto estremamente significativo. Non va certamente dimenticato come le scoperte scientifiche, che erano state realizzate nei decenni precedenti, siano state immediatamente inglobate nelle forme del fare arte, utilizzandole in modo molto spesso provocatorio e irriverente.

"Preceduta dalle innovazioni ottocentesche (colori industriali, fotografia, cinema), fin dai primi anni del secolo XX si mette in moto una straordinaria serie di esperienze: il mito tecnologico del Futurismo; il rituale del caso nel Dada; lo scardinamento concettuale del ready made di Duchamp; [....${ }^{10}$ Tutto quello che una volta era l'oggetto artistico, una rappresentazione, un prodotto di circostanziati e storicizzati procedimenti tecnici viene decostruito, messo in crisi, rimosso. L'opera d'arte si trasforma: da statica ad animata, da unicum a ripetibile e riproducibile, da compiuta a instabile ed effimera, da immodificabile a processuale e manipolabile, da oggetto di contemplazione a oggetto di partecipazione. [...]. La tecnica tradizionale è rifiutata a favore di nuovi mezzi che possono includere qualsiasi elementi $[. .$.$] . L'opera esprime un'inarrestabile tendenza a$ non identificarsi più con un oggetto ma con un'esperienza, una relazione lega artista e osservatore [...].” (Bordini, 2011, pp.11-12)

È così che specifici aspetti legati alla creazione materiale delle realizzazioni hanno recuperato il loro valore, facendoci riflettere, da un punto di vista educativo, su quanto il contatto con strumenti, materie anche non consuete, possa certamente arricchire le proposte all'interno degli stessi servizi. Come ben ci ricorda il critico e artista Gillo Dorfles da sempre: "L’opera d'arte, [...], risulta strettamente legata al suo medium- al

10 Qui lo scritto di Silvia Bordini elenca una lunga serie di cambiamenti che hanno potentemente influito nella trasformazione delle pratiche artistiche, si debbono tralasciare per la necessità di rispettare i limiti in termini di battute di questo contributo. 
suo mezzo espressivo- condizionata dai materiali in cui è realizzata, inseparabile dunque dalla stessa costituzione materiale e formale." (Dorfles, 2004, p.15) Aspetto evidenziato anche dallo stesso John Dewey, che ne parla anche da un punto di vista educativo, per le ricadute che questa prospettiva può avere nella progettazione delle azioni da proporre ai bambini: "In ogni opera d'arte, [...], questi significati sono in realtà incorporati in un materiale che diventa così il mezzo per la loro espressione.” (Dewey, 1995, p.274)

Sul contemporaneo è utile, però, inserire una precisazione, anche in questo caso riferendoci al pensiero di alcuni autori. Al di là delle specificità della nostra epoca, infatti, va segnalato come da sempre ogni opera sia stata contemporanea al suo tempo, come afferma il filosofo Jean-Luc Nancy: "L'arte, infatti, è sempre stata contemporanea al suo tempo: Michelangelo, Prassitele, il pittore di Lascaux sono tutti contemporanei dei propri contemporanei." (Nancy, 2007, p.3) Riferimento rintracciabile anche in Vittorio Sgarbi, critico d'arte, che ci apre verso un successivo passaggio:

"Conviene ribadire due concetti fondamentali e apparentemente contraddittori: 1) tutta l'arte è arte contemporanea; 2) contemporaneo è un dato non ideologico, ma semplicemente cronologico. È questa la forza dell'arte in divenire, che va ritenuta contemporanea non in quanto più o meno sperimentale, più o meno avanzata, ma solo in quanto concepita, elaborata ed espressa nel nostro tempo. Non c'è altro modo di essere contemporanei che essere qui e ora. Così, insieme alla contemporaneità di ciò che esiste, c'è la contemporaneità di ciò che è esistito e continua a vivere." (Sgarbi, 2012, p.29)

Anche se, va precisato, come con il termine "contemporaneo" molto spesso si voglia definire una proposta, un'azione, la pratica di un artista che non è totalmente appiattito sul presente, ma che presenta un pensiero che, pur radicandosi nel proprio tempo, riesce a travalicarlo, a superarlo o a collegarsi a tensioni anche passate o future: "Coloro che coincidono troppo pienamente con la loro epoca, combaciando perfettamente con essa in ogni punto, non sono contemporanei perché, proprio per questo, non riescono a vederla. [...] contemporaneo è chi riceve in pieno viso il fascio di tenebra proveniente dal suo tempo" (Agamben, 2008, p.15) non essendosi cioè fatto accecare dalla luce del presente, ma avendola affrontata, scrutata e avendo individuato nuove forme attraverso le ombre. Anche Joris-Karl Huysmann, tra gli altri, molto tempo prima ci aveva parlato degli artisti come di coloro che sanno stare sul ciglio del tempo (1908).

Allora ecco una proposta che può coinvolgere anche le istituzioni educative, quella di vivere in contatto con le forme di espressione artistica che le sono contemporanee, conoscendole, frequentandole come educatori e insegnanti ${ }^{11}$ e cogliendo nella messe così rigogliosa delle proposte nazionali, come pure internazionali, quegli spunti, quelle proposte che possono essere significativi anche all'interno dei contesti educativi.

\section{Arte come forma di comunicazione anche politica}

Se ci rivolgiamo, infatti, all'ambito del contemporaneo è ormai da molto tempo che in questo campo si parla di: installazioni, environment, ambienti che percorsi dal fruitore permettono di sperimentare

${ }^{11} \mathrm{Ci}$ si riferisce con questo anche alla frequentazione di musei, di siti culturali legati a qualsiasi proposta. Troppo spesso nei nidi e nelle scuole dell'infanzia italiane, come in quelle estere la proposta figurativa passa per l'apporto delle immagini edulcorate legate ai personaggi della Walt Disney, rifatti dai docenti. Sul rapporto con i musei si veda anche: Nardi, 1999; Panciroli, 2015; Zuccoli, 2010, 2014. 
una propria personale esperienza e dove la partecipazione e l'interpretazione del pubblico è richiesta e incentivata. Un'arte che sia solo da osservare, di cui ascoltare una spiegazione definita, non è quella verso cui attualmente si stanno muovendo artisti, musei, luoghi della cultura. Si chiede, invece, a gran voce una partecipazione, una presa di posizione. Con questo aspetto ci si riferisce all'arte intendendola anche come "arte relazionale", da una definizione coniata da Nicolas Bourriaud, che ha riscosso nel tempo molto successo. Egli ci parla, infatti, di una relazione che s'instaura tra artisti e fruitori in un gioco costante e mutevole, che prevede una continua trasformazione: "L'attività artistica costituisce un gioco le cui forme, modalità e funzioni evolvono, secondo le epoche e i contesti sociali; non è un'essenza immutabile." (Bourriaud, 2010, p. 11) Partecipare all'attività culturale vuol dire anche prendere posizione, individuare le proposte con cui ci si vuole confrontare con maggiore piacere, incontrare però allo stesso tempo quelle che invece suscitano rabbia, fastidio, pure in questo caso non lasciando ad altri di scegliere in che posizione metterci, e in questo interpretando l'arte per il suo portato politico e comunicativo. In questo contributo si vuole riferire, a titolo esemplificativo, di un caso di ricerca-azione, realizzato con la Fondazione Triennale di Milano, luogo espositivo legato in particolare al design, e l’Università di Milano Bicocca, Dipartimento di Scienze Umane per la Formazione, in relazione all'esposizione: La Terra Inquieta, ideata e curata da Massimiliano Gioni, prodotta in collaborazione tra Fondazione La Triennale e Fondazione Trussardi, che si è occupata, attraverso le opere d'arte, di uno dei temi più caldi della nostra società attuale, quello delle migrazioni, proponendoci un percorso. Come si può leggere nel catalogo:

"La Terra Inquieta prende a prestito il titolo da una raccolta di poesie dello scrittore caraibico Édouard Glissant, autore che ha dedicato la sua intera opera all'analisi e alla celebrazione della coesistenza di culture diverse. L'esposizione racconta il presente come un territorio instabile e in fibrillazione: una polifonia di narrazioni e tensioni. Attraverso le opere di più di sessanta artisti provenienti da oltre quaranta paesi del mondo - tra cui Albania, Algeria, Bangladesh, Egitto, Ghana, Iraq, Libano, Marocco, Siria e Turchia - e con l'inclusione di documenti storici e oggetti di cultura materiale, la mostra parla delle trasformazioni epocali che stanno segnando lo scenario globale e la storia contemporanea, focalizzandosi in particolare sulla rappresentazione della migrazione e della crisi dei rifugiati. La Terra Inquieta esplora geografie reali e immaginarie, ricostruendo l'odissea dei migranti e le storie individuali e collettive dei viaggi disperati dei nuovi dannati della terra." (Gioni, 2017, p.250)

La tematica affrontata da questa esposizione, così cruciale ai giorni nostri, benché non si dimenticasse della cronaca, si concentrava sull'arte, mettendo in scena le voci degli artisti. Alcuni di questi erano stati testimoni diretti degli eventi, altri, invece, si mostravano come potenti elaboratori delle esperienze contemporanee, capaci, con le loro creazioni, di raccontare, far vivere in prima persona ai visitatori: passaggi esperienziali, ricordi, reportage, storie, sogni, narrazioni, progetti, metafore svelando così un ruolo cruciale che l'arte può giocare ancora ai nostri giorni nel guardare con occhi diversi l'attualità, ma anche nel proporre ipotesi di trasformazione del mondo. L'artista, con la sua parola-pensiero concretizzata in un'opera di qualsiasi forma essa sia, diviene allora un testimone significativo, che ha bisogno però necessariamente di un confronto, di un dialogo anche acceso con il pubblico (Gadamer, 1986, pp.150-151; Eco, 1962). 
In questo caso insieme alle referenti dell'Ufficio Servizi al Pubblico e Ricerche della Triennale di Milano, Valentina Barzaghi e Valeria Marta, a partire dalla mostra La Terra Inquieta con l'apporto dell'Università, si sono progettati percorsi per ragazzi delle scuole secondarie di primo grado. Due le prime domande che si sono poste alla nostra attenzione, come gruppo di ricerca: come e se gli artisti con le loro produzioni uniti a uno specifico taglio ideativo e curatoriale potessero sollecitare, stimolare, arricchire e approfondire un dibattito? E come altresì l'opera, o una mostra, che qui si proponeva in un parallelismo con un coro polifonico e orchestrato delle singole opere, potessero essere vissute, non solo come pretesto (Dallari in Francucci, Vassalli, 2005) per affrontare una tematica, ma anche come interlocutori diretti (Cimoli, 2017) dei visitatori? Queste questioni iniziali ci hanno permesso di riflettere su quanto un differente canale comunicativo e una pluriformità di punti di vista, di strumenti e di proposte artistiche riesca a sollevare una coltre a volte ammantata da una pigrizia mentale abituata alla prolificità e superficialità di tante immagini e parole veicolate nei più abituali format comunicativi.

Da un punto di vista educativo ci si è interrogati anche su: che cosa scegliere? Con quali parole presentarlo? Attraverso quali interrogativi stimolare una partecipazione, un dibattito? Proponendo quali azioni permettere l'emersione e la sedimentazione di alcuni pensieri? Insieme a queste domande, come ben ci ha insegnato Bruno Munari (1981a, 1981b, 1984), e insieme a lui Franco Frabboni (2004) e Quinto Borghi (2003), si poneva una riflessione mirata anche sulla pratica laboratoriale che si sarebbe operata, come mezzo potente per mettere a tema praticamente le sollecitazioni che si sarebbero vissute attraverso la mostra.

Questo lavoro, seppur mirato a un'età più avanzata, permette di riflettere sul fatto che le proposte artistiche anche contemporanee possono risultare un valido ausilio per affrontare qualsiasi tipo di tematica, lavorando in molti modi, che qui si provano ad elencare: sui materiali, sui contenuti, sulle narrazioni, sulle biografie, sulle esperienze.

\section{Educazione all'arte come sperimentazione laboratoriale}

Come abbiamo visto nel secondo paragrafo, dedicato all'educazione all'arte in rapporto all'arte contemporanea, una parte fondamentale di questa disciplina è quella relativa alle pratiche, ai materiali e alla loro sperimentazione. Da sempre una grande attenzione è stata data alla componente materiale delle stesse opere, anche se le derive di un pensiero idealistico hanno cercato di far valorizzare esclusivamente l'idea, il pensiero, in particolare del genio, a discapito di tutta la parte operativa. La pratica laboratoriale, però, era sempre stata una modalità imprescindibile nel passato per pittori, scultori e artigiani, basti pensare alla bottega del Verrocchio dove lo stesso Leonardo si era formato. Nei secoli molti giovani erano cresciuti all'interno degli studi degli artisti più affermati, lavorando giorno dopo giorno nella preparazione delle tele, dei colori, dei materiali per la scultura e la decorazione. Si trattava dell'inizio di un percorso che non dimenticava mai la materialità. Questa modalità successivamente era stata modificata a favore della creazione delle scuole più codificate o delle Accademie di Belle Arti, dove, in ogni caso benché l'aspetto 
teorico fosse una parte certamente fondamentale, risultava sempre secondario rispetto alla sperimentazione costante delle tecniche e dei materiali. Va sottolineato come la volontà di mettere le mani in pasta, di misurarsi con la materia, sia, al di là della pratica artistica, una necessità stessa dell'essere umano, strettamente legata allo sviluppo della sua intelligenza e della sua possibilità di sopravvivenza.

Questo elemento imprescindibile, che tanto ha permeato e permeerà la storia dell'umanità oltre alle nostre proposte educative, ci viene meravigliosamente descritto da Richard Sennet nel testo "L'uomo artigiano"12. In queste pagine egli riscatta l'operatività umana da quel ruolo secondario in cui è stata rinchiusa per centinaia di anni, lavorando sul tema della tecnica: “[...] ma la tecnica considerata non come procedimento svincolato dal pensiero, bensì come questione culturale [...]”. (Sennet, 2014, p.18) L'autore proprio su questo argomento ci propone due tesi:

"[...] la prima che tutte le abilità, anche le più astratte, nascono come pratiche corporee; la seconda, l'intelligenza tecnica si sviluppa attraverso le facoltà dell'immaginazione ${ }^{13}$. La prima tesi pone l'accento sulla conoscenza acquistata nella mano attraverso il tatto e il movimento. La seconda argomentazione inizia analizzando il linguaggio che cerca di dirigere e di guidare l'abilità corporea. Tale linguaggio è più efficace quando mostra in modo immaginativo come si fa una certa cosa. L'uso di strumenti imperfetti o parziali stimola l'immaginazione a elaborare la capacità di riparare e improvvisare.” (Sennet, 2014, p.19)

L'aspetto corporeo, della pratica fisica, unito alla necessaria progettualità che nasce dalla resistenza e dalla difficoltà, si rivelano dunque pratiche istruttive di cui i servizi educativi e le scuole devono necessariamente fare tesoro. Proposte e lavori in cui la difficoltà e l'incertezza dei risultati portino i bambini a sperimentare e ad attivare l'immaginazione divengono elementi importanti nella progettazione di situazione di scoperta e apprendimento.

\section{Atelier e laboratori tra nido e scuola dell'infanzia}

In questo punto della trattazione è allora necessario inserire un collegamento alla pratica delle scuole di Reggio Emilia (Reggio Children $囚)$, che ha scelto fin dai tempi remoti dei suoi inizi di tenere in grande considerazione l'aspetto artistico ed estetico, coinvolgendo gli atelieristi. Queste specifiche professionalità formate nel mondo dell'arte in forte accordo con gli educatori e le comunità scolastiche si sono sempre occupate di permettere che le proposte dei bambini trovassero una loro voce e una loro concretizzazione, sviluppando progetti all'interno dei nidi e delle scuole. "L'atelier non è mai stato considerato come un luogo separato e specialistico dal resto della scuola: da subito il linguaggio visivo ha partecipato e si è intrecciato con tutti i campi del sapere”. (Vecchi in Edwards, Gandini, Forman, 1995, , p.138) Si tratta di una scelta potente che ha reso riconoscibile un progetto educativo e che ha permesso di dialogare con altre entità, con la garanzia del pieno sviluppo del valore della sua proposta:

"Come una possibilità di avere più punti di vista e dove il bello, la scelta estetica, non vengono considerati un optional ma una necessità del pensare e del vivere. Come contributo alla rottura di vecchi schemi pedagogici, rendendo attive le mani, la testa e le emozioni dei bambini e degli insegnanti. Come possibilità di rendere visibili alcune delle 12 La dimostrazione dell'attualità di questo testo si conferma anche dalle nuove pubblicazioni che riflettono, anche se con tagli
diversi, sulle medesime questioni, si veda almeno: Boncinelli e Sciarretta, 2015 .

13 Sul valore dell'immaginazione si guardi anche il testo di Bruno Munari Fantasia del 1977. 
grandi capacità dei bambini. [...] Io e le insegnanti lavoravamo spesso a specchio, imparando le une dalle altre ed entrambe dai bambini. In questo modo cercavamo assieme- atelieriste, insegnanti, bambini, pedagogisti- le strade di una nuova didattica dove il linguaggio visivo, interpretato, veniva intrecciato agli altri linguaggi, a loro volta interpretati, costruendo mappe di conoscenza dell'apprendimento del bambino e dell'insegnante." (Vecchi in Edwards, Gandini, Forman, 1995, pp.137-138).

Un altro aspetto da non dimenticare è l'uso specifico, anzi i plurimi usi, che il disegno assume in queste scuole, non solo come aspetto comunicativo, linguaggio precipuo dei bambini nei primi anni di vita, ma come modalità per interrogare la realtà, per vedere e capire il mondo. Per questo si inserisce in chiusura di questo paragrafo un breve scambio comunicativo tra due bambine, molto piccole, a proposito della scoperta delle ombre ${ }^{14}$ :

"Elisa F. e Elisa M. stanno disegnando se stesse con la propria ombra. -Si può controllare fuori se abbiamo fatto giusto. -Secondo me mi sono sbagliata un pochino: la mia ombra vera è tutta nera e attaccata ai piedi. -Devi metterla anche con la testa in giù e anche un po' di fianco. -Ma non vedi che l'hai fatta troppo lontana? Non so perché non fai i piedi attaccati. -Uno fa poi come vuole; io posso anche ritagliarla e metterla più vicino -Non puoi fare come vuoi; devi fare come vuole l'ombra -Il sole è più facile: vuole dall'altra parte del bimbo, lo so già! Edwards, Gandini, Forman, 1995, pp.166-167).

\section{Senza dimenticarsi della documentazione}

L'ultimo passaggio che si vuole sottolineare relativo al rapporto dell'educazione con l'arte è anche quello che si occupa della predisposizione degli spazi e dei materiali oltre che della documentazione. Anche in questo aspetto l'apporto dell'adulto con le sue scelte culturali e estetiche è fondamentale. L'incoraggiamento verso la sperimentazione, la riflessione, la cura nella scelta di materiali e strumenti con la scommessa sulle possibilità di azioni dei bambini sono dei passaggi che rendono significativo questo lavoro. Per renderli evidenti anche ai bambini delle altre sezioni, agli altri educatori, alla comunità ecco che la scelta di usare delle forme di documentazione che si ispirano a quanto l'arte contemporanea ci propone diviene una soluzione innovativa che garantisce un rapporto costante con la realtà che ci circonda (Vecchi, Giudici, 2015). Sentiamo ancora su questo punto l'esperienza di Reggio Emilia:

"Un'altra impronta rilevante, data dalla presenza dell'atelier, è stata la visibilità del progetto di lavoro realizzato, la documentazione era vista soprattutto come possibilità democratica di partecipazione dei bambini, dei colleghi, dei genitori, della città al progetto educativo. [...] La documentazione visiva è cresciuta nel tempo, diventando una pista argomentativa e narrativa molto efficace, che ci permetteva di rendere più visibile l'immagine complessa e competente del bambino e comunicare proposte didattiche innovative [...]."(Vecchi in Edwards, Gandini, Forman, 1995, pp.137-139).

14 Si tratta di un percorso progettuale molto più lungo che aveva coinvolto le sezioni, di cui qui si rendono solo alcuni passaggi comunicativi tra i bambini. 


\section{Conclusioni}

In questo breve scritto abbiamo provato a vedere come la storia e gli intenti politici abbiano nel tempo segnato le possibilità del fare educazione all'arte anche con i bambini più piccoli. L'idea che si ha dei bambini, delle loro potenzialità, come pure della stessa arte hanno un'influenza determinante sulle azioni educative che vengono progettate e realizzate negli spazi educativi. Se l'arte viene pensata con limitate possibilità, strettamente riproduttive, senza permettere variazioni di significati e di modalità operative, senza utilizzarla cioè rispettando il suo statuto epistemologico, anche le potenzialità dei bambini saranno concepite solo come esecutive e lineari, spegnendo gran parte delle risposte. Un contatto con le proposte del mondo dell'arte contemporanea, con le sue modalità di ricerca (Sullivan, 2005) possono invece risultare una delle possibili strade da percorrere per innovare questa disciplina. L'arte stessa, così intesa, con la partecipazione attiva di educatori e insegnanti, può diventare una forma comunicativa e politica di grande spessore. Di conseguenza le proposte scelte anche per i più piccoli si arricchiscono di mille potenzialità (Dallari, 2005) che non dimenticano il lato materiale, sperimentale, oltre che il valore necessario della ricchezza di una buona documentazione, che riesce comunicare a tutti i piccoli passi e le grandi scoperte nei territori della conoscenza.

\section{Referências}

AGAMBEN, Giorgio. Che cos'è il contemporaneo? Roma: Nottetempo, I Sassi, 2008.

BETTINI, Francesco. I programmi di studio delle scuole elementari dal 1860 al 1945. Brescia: La Scuola, 1950.

BONCINELLI, Edoardo e SCIARRETTA, Galeazzo. Homo faber. Storia dell'uomo artefice dalla preistoria alle biotecnologie. Milano: Baldini\&Castoldi, 2015.

BORDINI, Silvia (a cura di). Arte contemporanea e tecniche. Materiali, procedimenti, sperimentazioni. Roma: Carocci, 2011.

BORGHI, Battista Quinto. I laboratori extrascolastici. L’atelier espressivo. Azzano San Paolo: Edizioni Junior, 2003.

BOURRIAUD, Nicolas. Estetica relazionale. Milano: PostmediaSrl, 2010.

CIMOLI, Anna Chiara (a cura di). Che cosa vedi? Musei e pubblico adolescente, Busto Arsizio: Nomos edizioni, 2017.

DALLARI, Marco. L'arte per i bambini. In: FRANCUCCI, Cristina e VASSALLI, Paola, Educare all'Arte. Milano: Mondadori Electa, 2005, pp. 17-25. 
DEWEY, John. Arte come esperienza e altri scritti. Firenze: La Nuova Italia, 1995.

DORFLES, Gillo. Le oscillazioni del gusto. L'arte d'oggi tra tecnocrazia e consumismo. Milano: Skira, 2004.

ECO, Umberto. Opera aperta. Forma e indeterminazione nelle poetiche contemporanee. Milano: Bompiani, 1962.

EDWARDS, Carolyn, GANDINI Lella, FORMAN George (a cura di). I cento linguaggi dei bambini. L’approccio di Reggio Emilia all'educazione dell'infanzia. Bergamo: Edizioni Junior, 1995.

EFLAND, Arthur. A History of Art Education. Intellectual and Social Currents in Teaching the Visual Arts. New York. Teachers College Press, 1990.

FRABBONI, Franco. Il laboratorio. Roma-Bari: Laterza, 2004.

GADAMER, Hans-Georg. L'attualità del bello. Studi di estetica ermeneutica. Genova: Marietti, 1986.

GIONI, Massimiliano (a cura di). The Restless Earth, catalogo della mostra a cura di Milano: Mondadori Electa, 2017.

HUYSMANS, Joris Karl. Certains. Paris: Plon, 1908.

LOMBARDI, Franco V. I programmi per la scuola elementare dal 1860 al 1955. Brescia: La Scuola, 1987.

MAZZA, Ermanno. Incontrare l'immagine, per sapere e per fare. Prospettive didattiche. Roma: Anicia, 2001.

MUNARI, Bruno (a cura di). Il laboratorio per bambini a Brera. Bologna: Zanichelli, 1981 (cit. 1981a).

MUNARI, Bruno (a cura di). Il laboratorio per bambini a Faenza, al Museo Internazionale delle Ceramiche. Bologna: Zanichelli, 1981 (cit. 1981b).

MUNARI, Bruno (a cura di). I laboratori tattili. Milano: Zanichelli, 1984.

NARDI, Emma. Un laboratorio per la didattica museale. Formello (Roma): Edizioni Seam, 1999. 
NANCY, Jean-Luc, L'arte, oggi. In NANCY, Jean-Luc Nancy, DIDI-HUBERMAN, Georges, HEINICH, Nathalie \& BAILLY, Jean-Christophe. Del contemporaneo. Saggi su arte e tempo. Milano: Bruno Mondadori, 2007, pp.1-20.

PANCIROLI, Chiara. Le arti visive nella didattica. Teorie, esperienze e progetto dalla scuola dell'infanzia alla scuola secondaria. Verona: QuiEdit, 2012.

PANCIROLI, Chiara. Formare al Patrimonio nella scuola e nei musei. Verona: QuiEdit, 2015.

READ, Herbert. Educare con l'arte. Milano: Edizione di Comunità, 1962.

SGARBI, Vittorio. L'arte è contemporanea ovvero l'arte di vedere l'arte. Milano: Bompiani, 2012.

SENNET, Richard. L’uomo artigiano. Milano: Feltrinelli, 2014.

SIEGESMUND, Richard. Why do we teach art today? Conceptions of art education and their justification. Studies in Art Education. 1998, 39, 3, 197-214.

SULLIVAN, Graeme. Art practice as research. Inquiry in visual arts. Thousand Oaks, CA: Sage, 2005.

SWIFT, John. A manifesto for Art in Schools. Journal of art \& design education, 1999, v.18, iss1, pp.1-13.

TORNATORE, Lydia (a cura di). Arte e conoscenza. Torino: Loescher, 1982.

VECCHI, Vea e GIUDICI, Claudia (a cura di). Bambini arte artisti. I linguaggi espressivi dei bambini, il linguaggio artistico di Alberto Burri. Reggio Emilia: Reggio Children srl, 2004.

ZUCCOLI, Franca. Dalle tasche dei bambini... Gli oggetti, le storie e la didattica. Azzano San Paolo (Bg): Edizioni Junior, 2010.

ZUCCOLI, Franca. Didattica tra scuola e museo. Antiche e nuove forme del sapere. Parma: Edizioni Junior-Spaggiari, 2014. 